SHORT REPORT

\title{
Olfactory deficits and sleep disturbances in Parkinson's disease: a case-control survey
}

\author{
J M Henderson, Y Lu, S Wang, H Cartwright, G M Halliday
}

J Neurol Neurosurg Psychiatry 2003;74:956-958

Background: Olfactory and sleep disturbances are common in Parkinson's disease, and may be early disease indicators.

Objective: To obtain information about olfactory and sleep deficits preceding the onset of motor symptoms in Parkinson's disease.

Subjects: 38 community dwelling patients with Parkinson's disease (73\% response rate) and 32 age matched controls (60\% response rate).

Methods: Using a questionnaire survey, the frequencies, timing, and relations between olfactory and sleep disturbances, drug treatment, mood, and motor deficits in Parkinson's disease were compared with those in age matched controls. Reliability of information was validated by informant interview in $9 \%$ of the sample. Interdependency of factors was assessed using Fisher's fourfold table test, and differences between populations were analysed using $\chi^{2}$ and unpaired $t$ tests.

Results: Microsmia was reported by 26 patients (68\%) (and only one control), on average within a year of the diagnosis of Parkinson's disease. More patients than controls had excessive daytime somnolence $(45 \%$ v $6 \%)$, restless legs $(50 \% \vee 19 \%)$, and abnormal movements during sleep $(34 \% \vee 0 \%)$, which generally occurred three to five years after diagnosis and were independent of mood disorders and drug treatment.

Conclusions: Many patients with Parkinson's disease have microsmia at the onset of motor deficits, but some sleep disorders are a subsequent occurrence.

$\mathrm{D}$ iagnosis of Parkinson's disease is based on motor features (bradykinesia, rigidity, resting tremor), ${ }^{1}$ which occur after considerable dopaminergic cell loss. Identification of earlier clinical markers is paramount for success in putative preventative treatments. Recent reports suggest that non-motor abnormalities of sleep ${ }^{2}$ and olfaction ${ }^{3}$ may appear preclinically in Parkinson's disease. Furthermore, the proportion of early stage patients with olfactory dysfunction appears greater than of those with motor symptoms. ${ }^{4}$ In a survey of 61 patients, $48 \%$ talked during sleep and had nightmares or nocturnal restlessness before the diagnosis, but the exact timing was not examined. ${ }^{5}$ Another study reported that REM sleep behaviour disorder preceded Parkinson's disease by a median of three years. ${ }^{2}$

While several questionnaire based studies have examined the prevalence of sleep deficits in Parkinson's disease, ${ }^{6-8}$ there has been little study of the timing of the appearance of sleep and olfactory deficits in this disorder. We screened a community sample of patients with the disease and age matched controls to obtain further information about deficits preceding the onset of motor symptoms.

\section{METHODS}

We compiled and mailed a questionnaire to 105 consenting participants ( 52 Parkinson's disease patients and 53 controls) in the Parkinson's NSW brain donor programme at the Prince of Wales Medical Research Institute, Sydney. Patients with a clinical diagnosis of Parkinson's disease ${ }^{1}$ (made by a specialist neurologist) were selected. All but three were taking L-dopa. The controls had no history of neurological or psychiatric disease. No respondent had dementia (clinical dementia rating $>0.5$; most scored $>27 / 30$ on the mini-mental state examination score; three patients had mild cognitive impairment but all had scores of $>24) .{ }^{9}{ }^{10}$ All were community dwelling and most were living in their own homes.

Participants were asked whether they had any of following: excessive daytime sleepiness, suddenly falling asleep during the day, violent or disturbing nightmares, insomnia, restless legs, unusual movements or kicking during sleep, and a change in their ability to smell things. Each item required a yes or no response. The respondent was also asked to write the year the deficit began, and whether it was responsive to antiparkinsonian or other drugs.

The final dataset was based on completed questionnaires from 38 Parkinson's disease patients ( 17 men, 21 women; 73\% response) and 32 controls ( 11 men, 21 women; $60 \%$ response). Reliability of data was validated by informant interview in six subjects ( $9 \%$ of the sample). For presence or absence of specific deficits there was high agreement between patient and spouse for microsmia and depression (100\%) and for several sleep items ( $83 \%$ for insomnia, excessive daytime somnolence, restless legs, and disturbing or violent dreams); however, anxiety was more variable $(67 \%)$.

\section{Statistics}

Group demographic differences were assessed using unpaired $t$ tests, and group differences in frequencies of deficits were assessed using $\chi^{2}$ tests. A probability $(p)$ value of $<0.05$ was considered significant. To determine any relations between deficits within groups, dependency was tested between all possible pairs of: anxiety, insomnia, excessive daytime somnolence, restless legs, abnormal movements, and nightmares, using Fisher's fourfold table dependency test for variables with two categories. ${ }^{11}$ Results are reported as mean (SEM).

\section{RESULTS}

There was no difference between groups in age $\left(t_{68}=-1.3\right.$, $\mathrm{p}=0.21$ ) or prevalence of depression (table 1 ).

\section{Sleep disturbances}

Insomnia occurred in a comparable proportion of the controls $(11 / 32,34 \%)$ and patients (19/38, 50\%; p > 0.05). Excessive daytime sleepiness was seven times as common in Parkinson's disease patients as in controls $(p<0.001)$ and restless legs were twice as common $(p<0.02)$. One third of the patients who experienced restless legs considered L-dopa helpful, while excessive daytime sleepiness was not improved (table 
Table 1 Demographic, mood, and drug treatment data from the control and Parkinson's disease groups

\begin{tabular}{|c|c|c|}
\hline Variable & Controls & Parkinson's disease \\
\hline $\begin{array}{l}\text { Age (years) } \\
\text { Parkinson's disease duration (years) } \\
\text { Hoehn and Yahr score }\end{array}$ & $\begin{array}{l}74(2) \\
- \\
\text { Score } 0 \text { (normal) }\end{array}$ & $\begin{array}{l}72 \text { (1) } \\
8.2 \text { (1.0) } \\
1-1.5 \text { (mild), } n=5 \\
2-3 \text { (moderate), } n=17 \\
4-5 \text { (end stage), } n=8\end{array}$ \\
\hline \multicolumn{3}{|l|}{ Drug treatment } \\
\hline Total daily L-dopa dosage (mg) & - & $592(463)$ \\
\hline Total daily PDI dosage (mg) & - & $126(65)$ \\
\hline Duration of L-dopa (years) & - & $7.1(4.2)$ \\
\hline Use of sedatives/hypnotics & $3 / 32(9 \%)$ & $10 / 38(26 \%)^{*}$ \\
\hline Anxiety & $5 / 32(16 \%)$ & 19/38 (50\%)* \\
\hline Duration of anxiety (years) & $18.3(14.4)$ & $4.9(1.0)$ \\
\hline Onset of anxiety relative to Parkinson's disease (years) & - & $1.3(1.2)$ \\
\hline Improvement of anxiety after antiparkinsonian treatment? & - & Yes in $4 / 19(11 \%)$; no in $14 / 19(74 \%)$ \\
\hline Depression & $2 / 32(6 \%)$ & $6 / 38(16 \%)$ \\
\hline Duration of depression (years) & Unknown & $1.5(1.3)$ \\
\hline Onset depression relative to Parkinson's disease (years) & - & $1.8(0.9)$ \\
\hline Improvement of depression affer antiparkinsonian treatment? & - & Yes in $1 / 6(17 \%)$; no in $4 / 6(67 \%)$ \\
\hline Excessive daytime sleepiness & $2 / 32(6 \%)$ & $17 / 38(45 \%)^{*}$ \\
\hline Duration of excessive daytime sleepiness (years) & ? & $3.6(0.8)$ \\
\hline Onset of excessive daytime sleepiness relative to Parkinson's disease (years) & - & 4.5 (1.2) \\
\hline $\begin{array}{l}\text { Improvement in excessive daytime sleepiness after antiparkinsonian } \\
\text { treatment? }\end{array}$ & - & Yes in $3 / 17(18 \%)$; no in $14 / 17(82 \%)$ \\
\hline Restless legs & $6 / 32(19 \%)$ & $19 / 38(50 \%)^{*}$ \\
\hline Duration of restless legs (years) & $8.2(4.7)$ & $2.7(0.5)$ \\
\hline Onset of restless legs relative to Parkinson's disease (years) & - & $4.9(1.1)$ \\
\hline Improvement of restless legs after antiparkinsonian treatment? & _- & Yes in $7 / 19(37 \%)$; no in $11 / 19(58 \%)$ \\
\hline Disturbing or violent dreams & $1 / 32(3 \%)$ & $4 / 38(11 \%)$ \\
\hline Duration of the dreams (years) & 2 & $4.2(4.0)$ \\
\hline Onset of dreams relative to Parkinson's disease (years) & - & $3.0(0.6)$ \\
\hline Improvement of the dreams after antiparkinsonian treatment? & - & Yes in $0 / 4(0 \%)$; no in $4 / 4(100 \%)$ \\
\hline Microsmia & $1 / 32(3 \%)$ & $24 / 38(68 \%)^{*}$ \\
\hline Duration of microsmia (years) & 7 & $6.7(5.3)$ \\
\hline Onset of microsmia relative to Parkinson's disease (years) & - & $1.2(1.5)$ \\
\hline Improved microsmia affer antiparkinsonian treatment? & - & Yes in $1 / 24(4 \%)$; no in $23 / 24(96 \%)$ \\
\hline
\end{tabular}

Data are mean (SEM). Proportions of groups manifesting a particular deficit are also given as (\%). Where dichotomous responses do not equal $100 \%$, this is because the remaining patients were unsure.

${ }^{*} \chi^{2}$ test, $p<0.05$.

PDI, peripheral decarboxylase inhibitor.

1). These symptoms appeared within five years after diagnosis (table 1). Less than $11 \%$ of all respondents experienced disturbing or violent dreams ( $p>0.05$, group comparison), whereas abnormal sleep movements were reported by $13 / 38$ patients $(34 \%)(v 0 \%$ of controls; $\mathrm{p}<0.001)$. These sleep deficits occurred in the first few years of the disease and did not improve after L-dopa (table 1).

Anxiety was reported by half the patients and one sixth of the controls $(p<0.01 ;$ table 1$)$, and three times as many patients took sedative or hypnotic drugs $(\mathrm{p}<0.05$; table 1$)$. Most variables were unrelated, except for insomnia and restless legs, and restless legs and abnormal sleep movements, which were significantly interrelated $(\mathrm{p}<0.05)$.

\section{Olfactory deficits}

Two thirds of the patients ( $v$ one control) complained of altered ability to smell $(\mathrm{p}<0.001$; table 1$)$. Though the information was purely qualitative, our patients experienced microsmia (decreased olfactory acuity) rather than anosmia (total loss of ability to smell), with a mean onset within a year of diagnosis. This symptom was largely unresponsive to L-dopa.

\section{DISCUSSION}

Other than motor, cognitive, and mood abnormalities, disturbances of sleep and olfaction are the other major, but often overlooked, deficits found in Parkinson's disease. They have recently been proposed as potential preclinical markers. ${ }^{23}$ Our data confirm that microsmia is quite common in early Parkinson's disease, while sleep disturbances generally occurred three to five years after diagnosis. These deficits were not particularly responsive to L-dopa treatment, suggesting that biochemical and pathological abnormalities other than nigrostriatal dopamine loss must be involved.

Our $67 \%$ response rate parallels another questionnaire based study of sleepiness involving 3391 adults, with a 70\% response rate. ${ }^{12}$ The slight female preponderance in respondents $(58 \%)$ probably reflects the composition of the targeted population (58\% female), but it was similar to that $(58 \%)$ reported in a study of health factors, sampling 9897 adults over the age of $65 .{ }^{13}$ We are thus confident of sampling in a similar manner to larger studies, and that we can make some meaningful comparisons to other published reports.

We confirm previous findings ${ }^{8}$ that insomnia is common in aged persons and is not disease specific. While early anxiety in Parkinson's disease may predispose to insomnia, in our survey these variables did not show interdependency, indicating that insomnia occurs for other reasons. Our data suggest that insomnia appears about five years after diagnosis, at a comparable time to excessive daytime sleepiness, restless legs, and abnormal movements. The latter sleep disturbances were not as prevalent in controls (table 1). In a study of multiple sleep latency in 27 patients with Parkinson's disease, pathological sleepiness was common but did not relate to measures of sleep architecture (total sleep time or sleep stage percentages). ${ }^{14}$ We confirm that insomnia and excessive daytime sleepiness are not interdependent in Parkinson's disease patients, and that there are no simple associations between excessive daytime sleepiness and the quality and quantity of the previous night's sleep. ${ }^{14}$ 
REM sleep behaviour disorder is increased in Parkinson's disease, ${ }^{515}$ and a recent study reported that 13 of 25 patients (52\%) developed it before the clinical diagnosis of Parkinson's disease. ${ }^{2}$ Fifty per cent of our sample of patients with Parkinson's disease reported restless legs, unlike a previous study in which restless legs occurred in only $3 \%$ of such patients. ${ }^{16}$ Our data suggest that this is a later feature of Parkinson's disease, which may help explain this discrepancy. Restless legs, insomnia, and periodic limb movements appear to occur in concert in Parkinson's disease (these features were interdependent), consistent with the proposal that periodic limb movements affect the majority of individuals manifesting restless legs. ${ }^{17}$ Importantly, none of our controls who complained of restless legs reported abnormal movements during sleep, and there is no reason to suspect that controls interpreted the question differently from Parkinson's disease patients. The combination of these features in Parkinson's disease is suggestive, but not conclusive of REM sleep behaviour disorder. ${ }^{15}$

There has been relatively little study of the timing of olfactory deficits in Parkinson's disease. The absence of correlations between odour detection and identification deficits with disease stage or duration, and their presence in untreated new patients, suggests that they occur early in the course of the disease. ${ }^{18-20}$ It is of relevance that the cortical nucleus of the amygdala, which has major olfactory connectivity, degenerates in Parkinson's disease. This non-dopaminergic degeneration appears to be an early feature and may underlie some of the early olfactory deficits and therefore explain why they are not responsive to L-dopa. ${ }^{21}$

In contrast, deficits in odour discrimination relate to disease severity, indicating that they may be secondary to ongoing degeneration, ${ }^{20}$ possibly associated with progressive loss of the anterior olfactory nucleus during the course of Parkinson's disease. ${ }^{22}$ Our survey found that $68 \%$ of patients were aware of olfactory deficits, which they reported as occurring early in the disease. In a previous study, $72 \%$ of patients with Parkinson's disease were unaware of a smell disorder before formal testing, and those who were aware of it had significantly lower olfaction scores, with $13 \%$ having anosmia. ${ }^{18}$ This discrepancy may relate to the wording and timing of questioning the patients. Most of our patients had moderate to severe disease and were therefore more likely to have become aware of the development of olfactory deficits during the disease course, the timing of which they could estimate retrospectively with the aid of their spouse or caregiver. The mean of one year for onset of microsmia after diagnosis, based on retrospective reports from our patient group, may indicate that more subtle deficits were present preclinically, because our reports were based on when the patients first noticed microsmia, not when it first developed. Furthermore, in another study the use of a test battery incorporating assessments of olfaction, wrist movement, and depression was able to identify abnormal performance in $22.5 \%$ of at risk first degree relatives versus only $9 \%$ of controls, ${ }^{23}$ suggesting that there are preclinical deficits in a proportion of people. These studies indicate that olfactory deficits may precede motor deficits in some patients with Parkinson's disease. ${ }^{34}$

\section{ACKNOWLEDGEMENT}

We would like to thank the patients and their families who participated in the survey.

\section{Authors' affiliations}

J M Henderson, H Cartwright, G M Halliday, Prince of Wales Medical Research Institute and University of New South Wales, Sydney, Australia

Y Lu, S Wang, Department of Neurology, 1 st People's Hospital, University of Shanghai, Shanghai, China

Competing interests: none declared

Correspondence to: Dr J M Henderson, Department of Pharmacology, Bosch Building, University of Sydney, NSW 2006, Australia; jasmine@med.usyd.edu.au

Received 18 March 2002

In final revised form 3 March 2003

Accepted 12 March 2003

\section{REFERENCES}

1 Gelb DJ, Oliver E, Gilman S. Diagnostic criteria for Parkinson's disease. Arch Neurol 1999;56:33-9.

2 Olson EJ, Boeve BF, Silber MH. Rapid eye movement sleep behaviour disorder: demographic, clinical and laboratory findings in 93 cases. Brain 2000;123:331-9.

3 Wolters EC, Francot C, Bergmans $P$, et al. Preclinical (premotor) Parkinson's disease. J Neurol 2000:247:103-9.

4 Doty RL, Bromley SM, Stern MB. Olfactory testing as an aid in the diagnosis of Parkinson's disease: development of optimal discrimination criteria. Neurodegeneration 1995;4:93-7.

5 Comella CL, Nardine TM, Diederich NJ, et al. Sleep-related violence, injury, and REM sleep behaviour disorder in Parkinson's disease. Neurology 1998:51:526-9.

6 Pappert EJ, Goetz CG, Niederman FG, et al. Hallucinations, sleep fragmentation, and altered dream phenomena in Parkinson's disease. Mov Disord 1999;14:117-21.

7 Shulman LM, Taback RL, Bean J, et al. Comorbidity of the nonmotor symptoms of Parkinson's disease. Mov Disord 2001;1 6:507-10.

8 Tandberg E, Larsen JP, Karlsen KH. A community-based study of sleep disorders in patients with Parkinson's disease. Mov Disord 1998; 13:895-9.

9 Folstein MF, Folstein SE, McHugh PR. "Mini-mental state": a practical method for grading the cognitive state of patients for the clinician. $J$ Psychiatr Res 1975;12:189-98.

10 Morris JC. The clinical dementia rating (CDR): current version and scoring rules. Neurology 1993;43:2412-14.

11 Krauth J. Distribution-free statistics. Amsterdam: Elsevier Science Publishers, 1990

12 Nugent AM, Gleadhill I, McCrum E, et al. Sleep complaints and risk factors for excessive daytime sleepiness in adult males in Northern Ireland. J Sleep Res 2001;10:69-74.

13 Walters SJ, Munro JF, Brazier JE. Using the SF-36 with older adults: a cross-sectional community-based survey. Age Ageing 2001;30:337-43.

14 Rye DB, Bliwise DL, Dihenia B, et al. FAST TRACK: daytime sleepiness in Parkinson's disease. J Sleep Res 2000;9:63-9

15 Eisensehr I, v Lindeiner $H$, Jager $M$, et al. REM sleep behavior disorder in sleep-disordered patients with versus without Parkinson's disease: is there a need for polysomnography? J Neurol Sci 2001;186:7-11.

16 Snider SR, Fahn S, Isgreen WP, et al. Primary sensory symptoms in parkinsonism. Neurology 1976;26:423-9.

17 Guilleminault C, Brooks SN. Excessive daytime sleepiness: a challenge for the practising neurologist. Brain 2001;124:1482-91.

18 Doty RL, Deems DA, Stellar S. Olfactory dysfunction in parkinsonism: a general deficit unrelated to neurologic signs, disease stage, or disease duration. Neurology 1988;38:1237-44.

19 Doty RL, Stern MB, Pfeiffer C, et al. Bilateral olfactory dysfunction in early stage treated and untreated idiopathic Parkinson's disease. J Neurol Neurosurg Psychiatry 1992; 55: 138-42

20 Tissingh G, Berendse HW, Bergmans P, et al. Loss of olfaction in de novo and treated Parkinson's disease: possible implications for early diagnosis. Mov Disord 2001;16:41-6.

21 Harding AJ, Stimson E, Henderson JM, et al. Clinical correlates of selective pathology in the amygdala of patients with Parkinson's disease. Brain 2002; 125:2431-45

22 Pearce RK, Hawkes CH, Daniel SE. The anterior olfactory nucleus in Parkinson's disease. Mov Disord 1995;10:283-7.

23 Montgomery EBJ, Baker KB, Lyons K, et al. Abnormal performance on the PD test battery by asymptomatic first-degree relatives. Neurology 1999;52:757-63.

24 Gonera EG, van $t^{\prime} \mathrm{H}$ of $M$, Berger HJC, et al. Symptoms and duration of the prodromal phase in Parkinson's disease. Mov Disord 1997; 12:871-6. 